\title{
Pressure Drop Correlation Covering Dilute to Dense Regimes of Solid Particle-Gas Flow in a Vertical Conveying Pipe
}

\author{
Y. Bindar, N.A. Sutrisniningrum \& D. Santiani \\ Energy and Processing System of Chemical Engineering Research Group \\ Chemical Engineering Program Study, Faculty of Industrial Technology \\ Institut Teknologi Bandung \\ Labtek X, Jl. Ganesha 10-Bandung 40132 \\ Email: yazid@che.itb.ac.id
}

\begin{abstract}
More general correlations between pressure drop and gas-solid flow variables are developed from the present experimental data. The correlation was modeled for a pneumatic conveying system in a vertical pipe. The transition boundary between dense and dilute regimes is constructed from the pressure drop correlations. The gas-solid particle flow variables are quantified by the gas Reynolds $\left(N_{r e f}\right)$ and the solid Froude $\left(F_{r p}\right)$ numbers. The dense flow regime is indicated by the decrease of the pressure drop with the increase of the gas Reynolds number. In contrary, the dilute regime exhibits the increase of the pressure drop with the gas Reynolds number. The proposed correlations were built at the range of gas Reynolds number ${ }_{f}$ from 360 to 500 and solid Froude number from 0,01 to 0,02 .
\end{abstract}

Keywords: dense phase; dilute phase; flow regimes; pneumatic conveying; pressure drop.

\section{$1 \quad$ Introductions}

Pneumatic conveying pipe is one of the methods for the solid particle transportation. Gases are used to carry the solid particle in the pipe. The carrier gas transfers its momentum to the solid particles. As a result, the particle flows together with the carrier. However, due to the friction between gas to particles, particles to -particles, gas to wall and particle to wall interaction, the amount of the energy to be supplied to the carrier gases is much higher than the gas flow only.

The friction in the piping line is indicated by the pressure drop that occurs between the inlet and the outlet of the transport pipe. Therefore, it is essential to predict accurately the total pipeline pressure drop, for example, if we can predict say, $\Delta p_{t}=800 \mathrm{kPa} \pm 25 \%$, the resulting uncertainty is too great (600 to $1000 \mathrm{kPa}$ ). This situation could lead to serious operating problems such as inadequate capacity and pipeline blockage.

Received March $22^{\text {nd }}, 2009$. 
Most existing models, such as reviewed [1] are suited only to pure dilute-phase or dense-phase applications. However, the possible modes of flow over long distances could occur between these two extremes such as dune-flow, sliding beds, and irregular slugging. The pressure drop correlations within such these transition regimes are rarely reported by authors.

The solid-gas transport in the pipeline must be able to be operated at wide range of flow regimes at safe condition and low maintenance cost. High maintenance cost usually occurs due to high friction factor of the gas-solid particle in which it contributes to higher power consumption. Another reason to high cost is shorter life time the ducting pipes that is caused by solid particle attrition, and abrasion [2].

Conveying the solid particle at dilute phase flow regime is a common method that is applied in industries. However, there are some advantages also to convey the solid particles in the dense phase flow regime. Paul [3] identifies those advantages as (i) decreased energy usage due to significantly reduced volumes of air, (ii) reduced material breakage or degradation due to slower conveying velocities, (iii) reduced pipeline wear due to lower conveying velocities, (iv) smaller conveying pipeline sizes due to heavier line loading capabilities and (v) smaller dust collection requirements at the material destination due to lower conveying air volumes.

It is our interest in this paper to formulate the pressure drop correlation by conducting the experiment. The correlation is applied for dense, transition and dilutes regimes of the solid particle gas flows. The result of this work shows that a general form of pressure drop correlation to cover all solid-gas flow regimes has been successfully developed.

\section{$2 \quad$ Fundamental}

To predict the behaviors of a gas-solid pneumatic system, the flow regimes and pressure drop, comprehensive characterization of the gas-solid flow is needed. With this information, the strategy to minimize power consumption can be set. For an example, the gas flow rate can be determined as minimum as possible to carry a required solid particle mass flow rate. At minimum gas flow rate, the attrition and abrasion can be also minimized.

One of the most prominent factors in pneumatic conveying is power consumption or power loss, which can be represented by the pressure drop exhibited by the system. In most cases, the pressure drop in the multiphase flow is higher than pressure drop in the one phase flow. According to Fan and 
Zhu [2], in the multiphase flow, pressure drop is caused not only by friction of gas molecules, but also by interaction between gas and solids, between solids itself, and external forces such as gravitational force, and electrostatic force. Those forces between solids and external forces cannot affect the gas motion directly, but it has effect on interaction system between gas and solid.

The transport characteristic of multiphase flow can be differentiated by the interaction pattern between molecules in the system. Two distinguished types of gas-solid particles flow pattern are dense-phase and dilute-phase flow [4]. Dilute-phase system occurs in the system with lower solid particles flow rate and higher gas flow rate than dense phase. In the dilute-phase flow, interaction between solid particles is insignificant compared to that in dense phase. According to Fan and Zhu [2], dilute and dense-phase can be identified by pressure drop to air velocity relation tendency. The solid-gas flow in the pneumatic conveying system is generally characterized by the relation between pressure drop to liner gas velocity. The relation is strongly influenced the solid particle mass flux in the flow, $G_{p}$. A typical form of the relation is described by Figure 1. The comparison between dense and dilute-phase characteristics is shown in the Table 1.

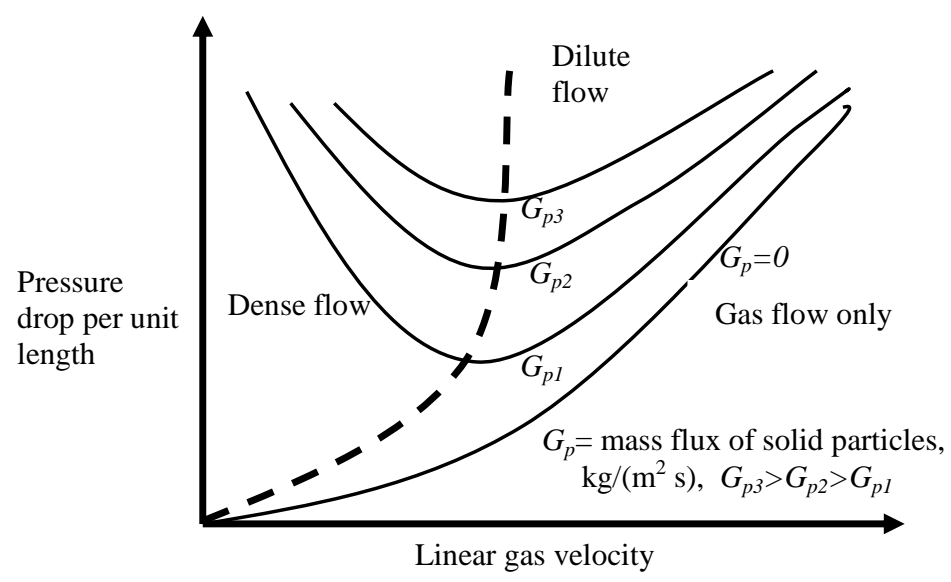

Figure 1 General pneumatic conveying system characteristic [2].

The gas-solid flow regimes in a vertical pipe are presented in the Figure 2. These flow regimes are defined by solid particles concentration, gas velocity, gas and solid particles properties and equipment orientation. The pressure drop at each regime is correlated to these variables.

Correlations to predict pressure drop in the gas-solid particles flow, acquired by former researcher, are presented in Table 2. These correlations only valid in the dilute-phase region and at certain flow range [1]. In this matter, only Khan and 
Pei correlation is considered to work under wide range of flow regimes. However, this correlation has not been able to arrive on exact situation for zero solid flow in which the pressure ratio value should be one.

Table 1 Comparison between dense and dilute-phase characteristic [5].

\begin{tabular}{lll}
\hline \multicolumn{1}{c}{ Criterion } & \multicolumn{1}{c}{ Dilute flow } & \multicolumn{1}{c}{ Dense Flow } \\
\hline $\begin{array}{l}\text { Relatif motion between } \\
\text { solid particles }\end{array}$ & High & Low \\
$\begin{array}{l}\text { Solid particles interaction } \\
\text { Perticle diffusivity }\end{array}$ & Weak & High \\
Viscosity & $\begin{array}{l}\text { Caused by gas and solid } \\
\text { particles interaction }\end{array}$ & $\begin{array}{l}\text { Low } \\
\text { Caused by solid } \\
\text { particles and gas to } \\
\text { solid particles } \\
\text { interaction }\end{array}$ \\
& & unstable \\
\hline $\begin{array}{l}\text { Motion over the minimum } \\
\text { transport velocity }\end{array}$ & Stable & \\
\hline
\end{tabular}

Dilute-phase

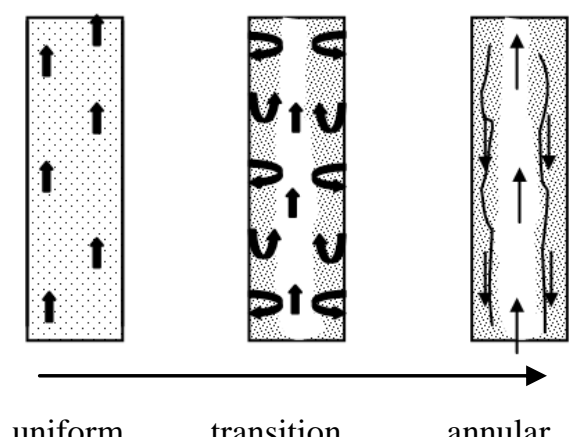

Dense-phase

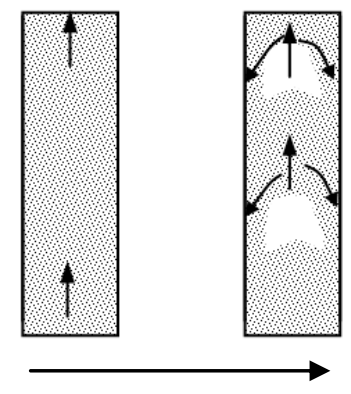

extrusion slugging

Decreasing gas velocity with constant solid particles concentration

Figure 2 Flow regimes of the gas-solid particles flow in a riser [4].

\section{Experimental Method and Apparatus}

This research was conducted by using sand solid particles. The gas carrier itself is air. Air is blown to a vertical observation pipe. The observation pipe has the sizes of $0.0504 \mathrm{~m}$ diameter and $0.775 \mathrm{~m}$ length. The equipment consists of a blower with $20 \mathrm{~m}^{3} / \mathrm{h}$ flow rate capability. The apparatus is equipped also with a solid particle Hooper-feeder, an observation pipe segment and a cyclone. The solid particle size ranges from 250 to $600 \mu \mathrm{m}$. The pressure drops and flow regime data were acquired at varied gas flow rate and solid particles flow rate. 
The equipment and the measuring devices are orificemeter, manometer and rotary valve. The experimental set-up is shown schematically by Figure 3 . The solid particles were sized with two standard screens for the sizes of 40 and 60 mesh. Before being used, the solid particles were dried to remove water vapor contents.

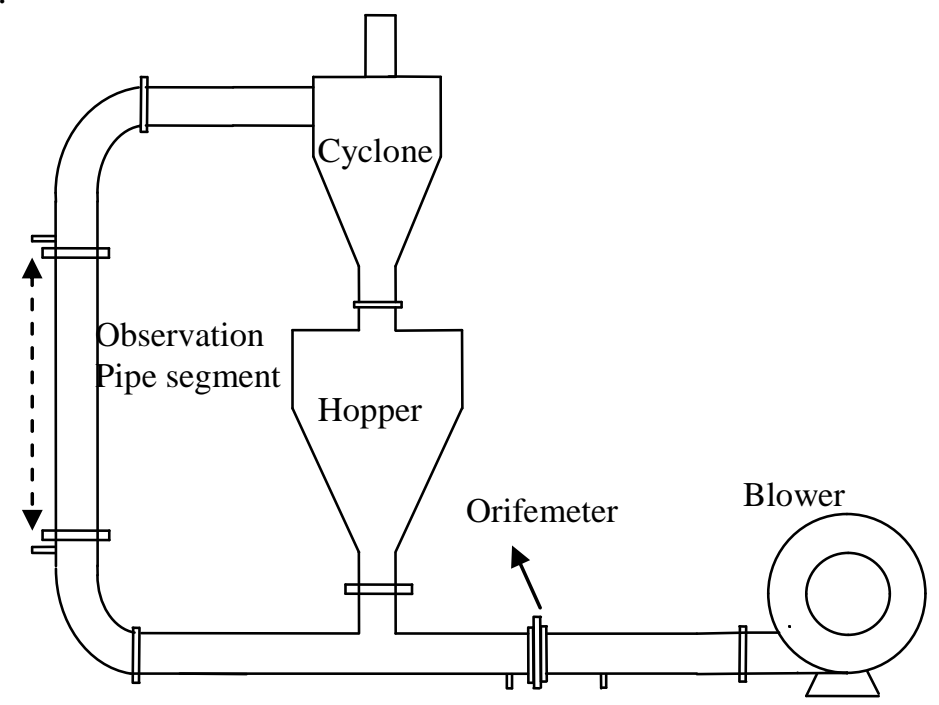

Figure 3 Experimental apparatus setup.

\section{Pressure Drop Correlation Development}

The pressure drop correlations were developed by transforming graphical data in Figure 4 to a mathematical model. The dependent variable in the model is the ratio between the pressure drop of gas-solid particles flow $(\Delta P)$ and pressure drop of gas flow without solid particles $\left(\Delta P_{f}\right), \Delta P / \Delta P_{f}$. The independent variables are Reynolds number of gas $\left(N_{\text {ref }}\right)$ and the solid particle Froude number $\left(\mathrm{F}_{\mathrm{rp}}\right)$. The Reynolds number is defined by

$$
N_{r e f}=\frac{U_{f} D \rho_{f}}{\mu_{f}}
$$

where $U_{f}, D, \rho_{f}$, and $\boldsymbol{\mu}_{f}$ are gas superficial velocity, pipe diameter, gas density and viscosity respectively. The solid particle Froude number $\left(F_{r p}\right)$ is calculated from

$$
F_{r p}=\frac{U_{p}^{2}}{g d_{p}}
$$


where $U_{p}, d_{p}$, and $g$ are the particle velocity, particle diameter and the gravity constant. The particle velocity is obtained from the particle mass flow rate, $\mathrm{m}_{\mathrm{p}}$, particle density $\rho_{p}$ and the pipe diameter as follows

$$
U_{p}=\frac{4 m_{p}}{\pi d_{p}^{2} \rho_{p}}
$$

These two variables represent gas and solid particle flow identifiers which influence directly the pressure drop for gas-solid particle flow.

Most intention in building the pressure drop correlation for solid-gas flow in pneumatic conveying pipe was paid to dilute phase flow regimes. Some of the correlation for dilute phase regimes is shown in Table 2 . The process variables that are involved in those correlation are fluid mass flux $G_{f}$, solid mass flux $G_{p}$, gas Reynolds number $N_{r e f}$, solid Froude number $F_{r p}$, pipe length $L$, pipe diameter $D$, pipe cross section area $A$, particle diameter $d_{p}$, fluid properties, solid properties, gravity constant $g$, pipe orientation angle $\boldsymbol{\alpha}$, drag coefficient $C_{D}$, and friction coefficient $f$.

Mathur and Klinzing [6] developed a pressure drop correlation for the dense phase regime as is shown by the following equation

$$
\frac{\Delta P}{L}=55.5 \frac{D^{0.1}}{U_{f}^{0.64} d_{p}^{0.26} \rho_{p}^{0.91}}(1-\varepsilon) \rho_{p} U_{f}^{2}
$$

where $\varepsilon$ is the gas volume fraction in the gas-solid flow in the pipe. Such this form of the model has not shown a general form that can be used in all regime condition.

The variables of fluid Reynolds number and solid Froude number already represent all geometry, properties and flow characteristics of fluid and solid. For this pressure correlation development, the dense phase regime is included. These two non-dimensional variables should also apply for dense phase regime as are shown for dilute phase.

Our experimental data behavior in Figure 4 presents a kind of decay function. This decay function might be represented by an exponential or power function. Actually, the function behavior has two boundary conditions. First, $\Delta P / \Delta P_{f}$ should be equal to one when $F_{r p}$ is equal to zero. Since there is no solid particles loaded at $F_{r p}=0$, the pressure drop of the gas-solid particle flow will same as pressure drop gas without solid particles at the same $N_{r e f}$. Second, the 
gas-solid particles flow resembles the fixed bed flow when the gas velocity approaches to zero.

According to the Ergun equation for fixed bed pressure drop model, at the dense-phase flow, $\Delta P / \Delta P_{f}$ should be diversely proportional to $N_{\text {ref }}$. While in the dilute-phase flow (high $\left.N_{r e f}\right), \Delta P / \Delta P_{f}$ approach to a constant value at a spesified value of $F_{r p}$. While in the dense-phase flow for $N_{r e f}$ approaching to zero, the value of $\Delta P / \Delta P_{f}$ become very large.

Some assumptions were applied for this model development. The voidage is uniform at any segment of the vertical pipe. The mass solid particle velocity is constant. The linear solid particle velocity is also constant at any segment.

Table 2 Pressure drop correlations developed by other authors for dilute phase [1].

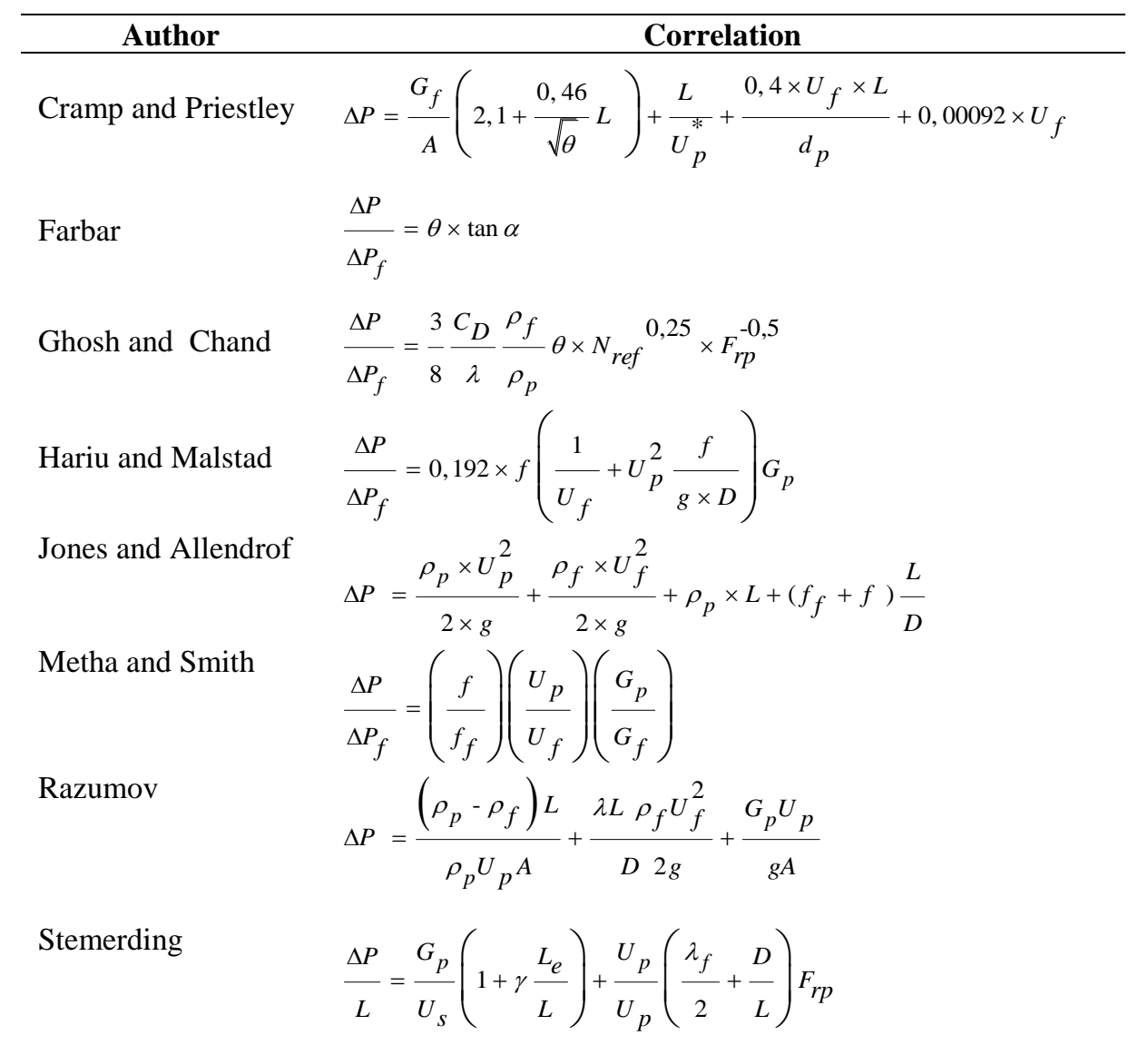




\begin{tabular}{ll}
\hline \multicolumn{1}{c}{ Author } & Correlation \\
\hline Vogt and White & $\frac{\Delta P}{\Delta P_{f}}=k\left(\frac{D}{d_{p}}\right)^{2}\left(\frac{\rho_{f}}{\rho_{p}} \frac{\theta}{N_{r e f}}\right)^{K}$ \\
Khan and Pei & $\frac{\Delta P}{\Delta P_{f}}=2,66 \times\left(\frac{C_{D}}{\lambda}\right)\left(\frac{\rho_{f}}{\rho_{p}}\right)\left(\frac{d_{p}}{D}\right)^{2}\left(\frac{N_{r e f}}{F_{r p}}\right)^{0,5} \theta \times \varphi^{-0,5}$ \\
\hline
\end{tabular}

Base on the the boundary approaching method, the pressure drop correlation is proposed as stated by Equation (5). The model is formulated to fulfill the boundary conditions specified above. The experimental data are used to fit this model. This model should work on all flow regimes.

$$
\frac{\Delta P}{\Delta P_{f}}=1+a F_{r p}\left(\frac{b}{N_{r e f}}+c\right)
$$

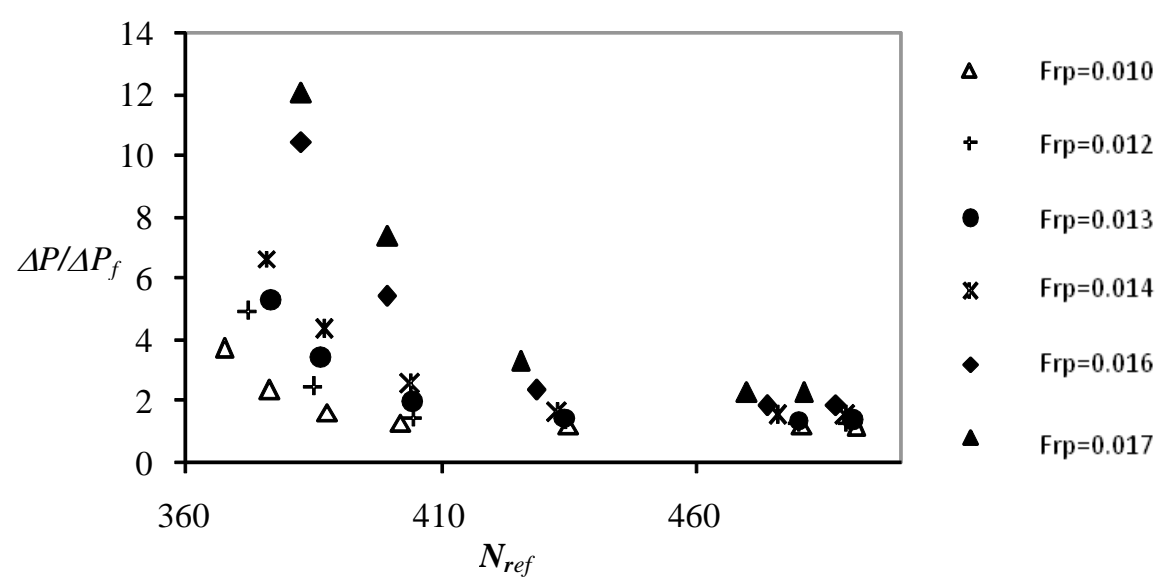

Figure 4 Pressure drop as a function of $N_{r e f}$ at various $F_{r p}$

\section{$5 \quad$ Result and Discussion}

Constants of the correlation, a, b, and, c of Equation (5) were obtained by fitting this model to the present experimental data. The model was fitted for dense and dilute phases. The best values of the model constants are given in Table 3 .

There are two types of flow regimes at linear gas velocity between $0.1-0.12$ $\mathrm{m} / \mathrm{s}$, slugging and annular. These are defined as the dense-phase flow. The slugging regime occurs when the linear gas velocity is smaller than that in the 
annular regime [4]. When the linear gas velocity is further decreased, the slugging mode become packed bed mode whereas solids tend to move slowly through the line with a density close to the bulk density of the material.

Table 3 Constants of developed model for each flow region.

\begin{tabular}{cccc}
\hline Regime & $\mathrm{a}$ & $\mathrm{b}$ & $\mathrm{c}$ \\
\hline Dense-Phase & $9.613 \times 10^{8}$ & -1630 & 8.751 \\
Dilute-Phase & $1.096 \times 10^{6}$ & -287 & 3.988 \\
\hline
\end{tabular}

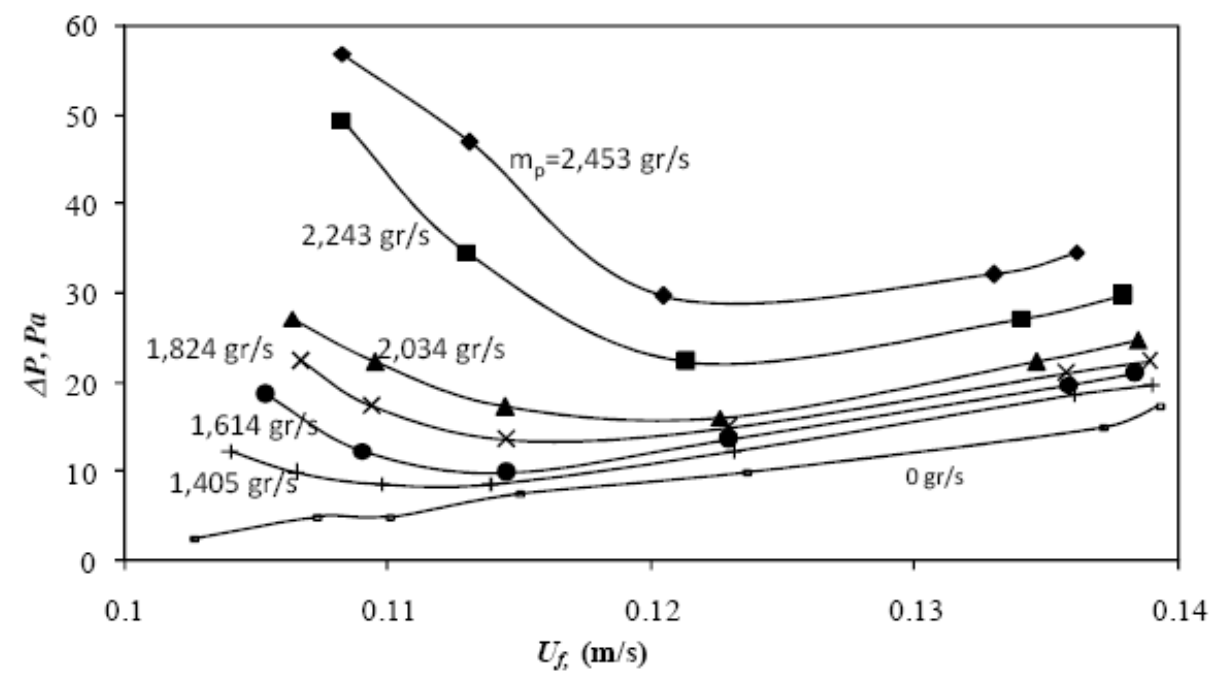

Figure 5 Relation between pressure drop with linear gas velocity at mass various solid particle velocity from the present experimental data.

The pressure drop in vertical conveying pipe is contributed by two components. They are wall frictional loss component and gravitational component, Fan and Zhu (1998). The present experimental data exhibit the relation between the solid gas flow pressure drops between the linear gas velocities, shown by Figure 5 . At high gas velocities (dilute-phase flow), pressure drop increases as gas velocity rises. It will increase the wall friction which is significantly higher than gravitational component. As the gas velocity is further reduced, the gravitational component becomes more significant.

Further decrease of the gas flow rate, the regime falls to the dense-phase flow. At this condition, the pressure drop is lifted up for higher solid flow rate. Moreover, the gravitational component becomes predominant. When the change of the pressure drop with the gas velocity becomes flat, the transition 
region between dense and dilute phases starts to occur. The wall friction and the gravitational force are both dominant.

The transition line between the dense and dilute phase regimes can then be defined. The line is formed by connecting the minimum pressure drop point at optimum gas velocity for each solid flow rate. Therefore, mathematically this minimum pressure drop is obtained from the pressure drop correlation of Equation (5) when the gradient of the pressure drop over the gas Reynolds number becomes zero at each value of Froude number as is stated by Equation (6).

$$
\left.\frac{\partial\left(\Delta P / \Delta P_{f}\right)}{\partial\left(N_{r e f}\right)}\right|_{F_{r p}}=0
$$

This line equation is used to predict the transition regime in term of the gas Reynolds number at specified solid particle Froude number. The transition regime line equation is then obtained as is defined by Equation (7).

$$
\begin{aligned}
\frac{\partial\left(\frac{\Delta P}{L}\right)}{\partial N_{r e f}}=0 & \frac{-1.3035 \times e^{\left(0.0056 \times N_{r e f}\right)} a \times b \times \ln \left(N_{r e f}\right) F_{r p}\left(\frac{b}{N_{r e f}}+c\right)}{N_{r e f}^{2}}+ \\
& \left.0.073^{\left(0.0056 \times N_{r e f}\right)}\left(1+a \times F_{r p}^{\left(\frac{b}{N_{r e f}}+c\right.}\right)\right)
\end{aligned}
$$

Table 4 Minimum pressure drop at $U_{f}$ minimum for every $F_{r p}$ at transition between dilute-phase and dense-phase flow.

\begin{tabular}{ccc}
\hline $\boldsymbol{F}_{r p}$ & $\boldsymbol{U}_{f}$ minimum & $\boldsymbol{\Delta P}$ minimum \\
\hline $9.97 \mathrm{E}-03$ & 0.113 & 8.86 \\
$1.15 \mathrm{E}-02$ & 0.114 & 10.50 \\
$1.29 \mathrm{E}-02$ & 0.115 & 12.15 \\
$1.44 \mathrm{E}-02$ & 0.121 & 18.42 \\
$1.59 \mathrm{E}-02$ & 0.122 & 21.50 \\
$1.74 \mathrm{E}-02$ & 0.123 & 28.46 \\
\hline
\end{tabular}


The minimum value of the pressure drop is given by the set of the gas velocity and solid particle Froude number. The results are presented in Table 4.

From the constructed model above, the flow regimes can be remapped from the present experimental data in a continuous nomograph. This constructed nomograph is shown by Figure 6. The model lines and the present experimental data fit closely.

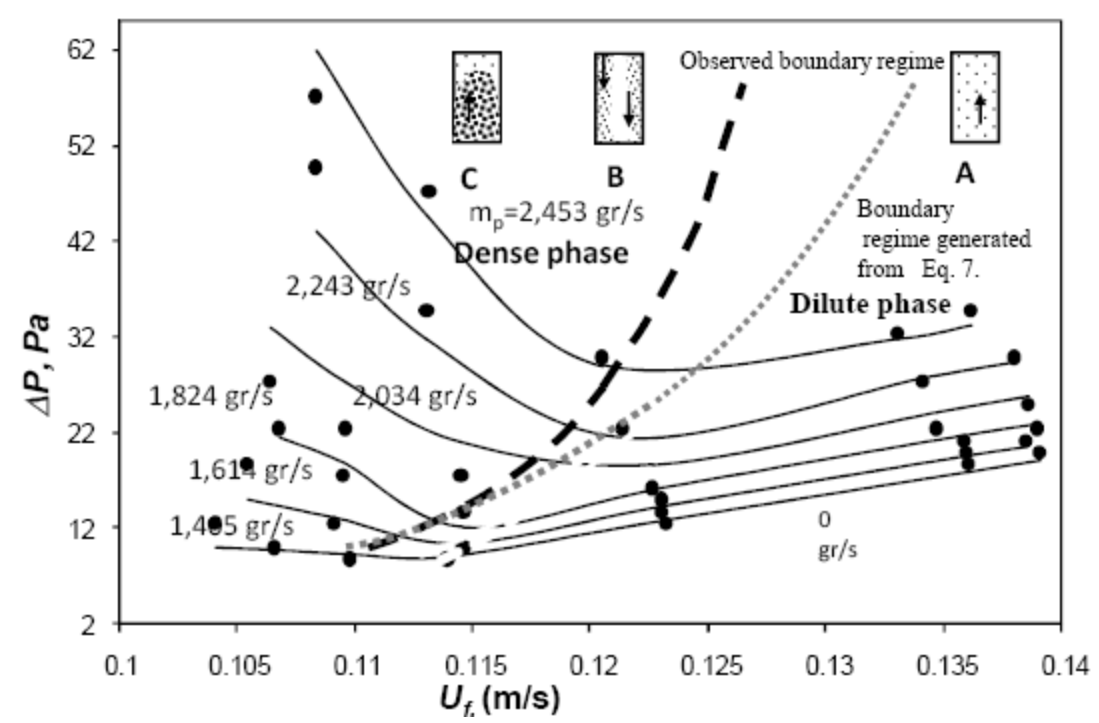

Figure 6 Nomograph of flow regimes map separated by a transitional line for a vertical pneumatic conveying system. The gas is air and the solid particles are sand particle.

The pipe diameter and length are 0.0508 and $0.775 \mathrm{~m}$ respectively. The solid points are the present experimental data. The solid lines are obtained from Equation (5) at solid flow rates $\left(\mathrm{m}_{\mathrm{p}}\right)$ in the range of 0 to $2.453 \mathrm{gr} / \mathrm{s}$.

\section{Conclusions}

The transition flow regimes of pneumatic conveying in a vertical pipe is successfully defined using the constructed pressure drop correlation. The present pressure drop correlations are considered more general, robust and valid to the boundary conditions. The transition line between dense and dilute regimes is stated in a mathematical equation form. A nomograph of the flow regime of solid-gas conveying flow from dilute to dense with the exact line transition is constructed using the present pressure drop correlation. 


\section{Notations}

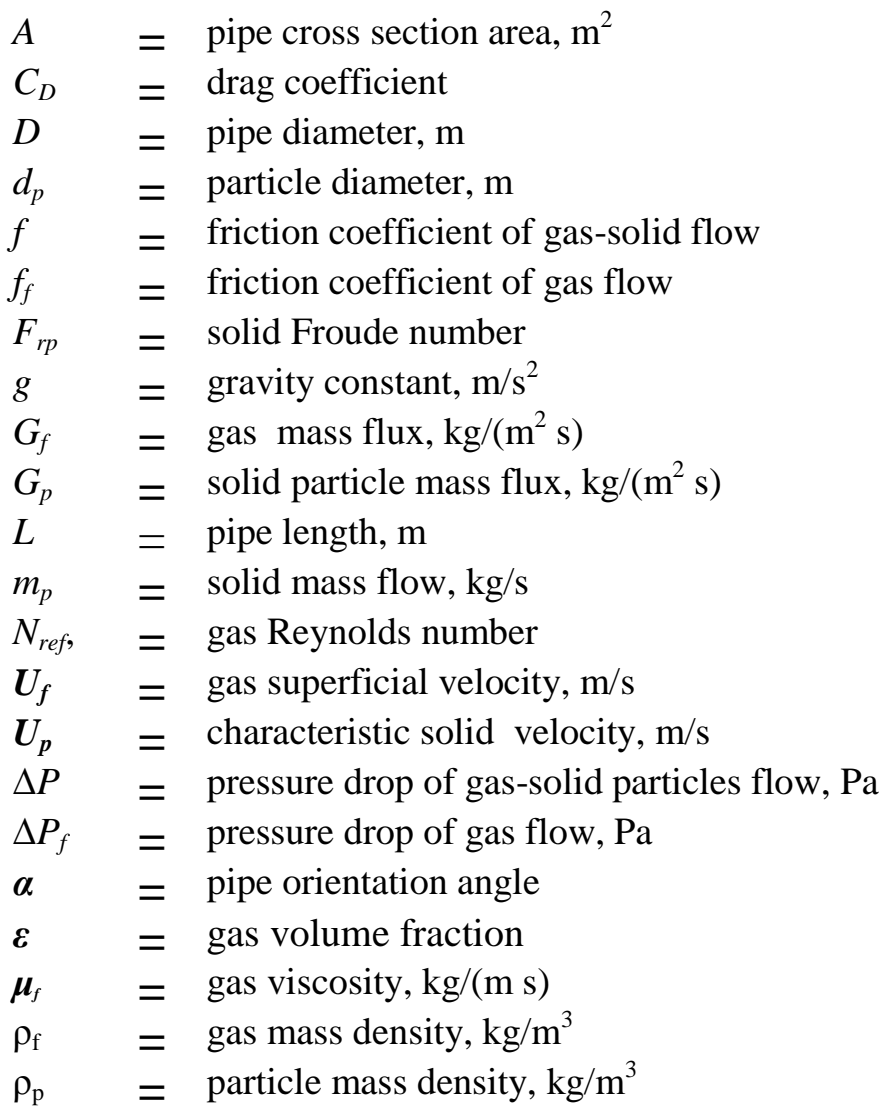

\section{References}

[1] Khan, J.I. \& Pei, D.C., Pressure Drop in Vertical Solid-Gas Suspension Flow, Ind. Eng. Chem. Process Des. Develop., 12(4), 1973.

[2] Fan, L.S. \& Zhu, C., Principles of Gas Solid Flows, Cambridge University Press, 1998.

[3] Paul, K.D., Dense Phase Pneumatic Conveying Improving Efficiency, Powder and Bulk Engr., p. 41, 1991.

[4] Cheremisinoff, N.P., Gas Flow Pocket Handbook, Gulf Publishing Co., Houston, 1984.

[5] Soo, S.L., Particulate and Continuum: Multiphase Gas Dynamics, Hemisphere, New York, 1989.

[6] Mathur, M.P. \& Klinzing, G.E., The Dense and Extrusion Flow Regime in Gas-Solid Transport, Can. J. of Chem. Engr., 59:590-594, 1981. 